\title{
SOME APPLICATIONS OF THEOREMS ON UNIFORM CAUCHY POINTS TO INFINITE SERIES ${ }^{1}$
}

\author{
C. W. MCARTHUR
}

1. Introduction. Uniform Cauchy points and points of uniform convergence of a sequence of functions are important in certain convergence questions. ${ }^{2}$ They will be applied here to obtain results on series in pseudo-metric semigroups by topologizing $P(N)$, the permutation group of the natural numbers $N$, and setting up the sequence of partial sum functions of the series as did Agnew [1] and Sengupta [8].

It has long been known that $P(N)$ is a metric space with the Fréchet metric defined by

$$
d_{F}\left(t, t^{\prime}\right)=\sum_{i=1}^{\infty} \frac{1}{2^{i}} \frac{\left|t(i)-t^{\prime}(i)\right|}{1+\left|t(i)-t^{\prime}(i)\right|}
$$

for each $t$ and $t^{\prime}$ in $P(N)$, and that if $\left\{t_{n}\right\}$ is a sequence in $P(N)$ and $t \in P(N)$, then $\lim _{n \rightarrow \infty} d_{F}\left(t_{n}, t\right)=0$ if and only if $\lim _{n \rightarrow \infty} t_{n}(i)=t(i)$ for each $i \in N$. If $X$ is a set and $d$ is a pseudo-metric for $X$ let $(X, \mathcal{T}(d))$ denote the topological space consisting of the set $X$ and topology $\mathcal{T}(d)$ where $\mathcal{T}(d)$ has the basis $[V(x, \epsilon): x \in X, \epsilon>0]$ where $V(x, \boldsymbol{\epsilon})$ $=\left[x^{\prime}: d\left(x, x^{\prime}\right)<\epsilon\right]$. Agnew [1] shows that $P(N)$ with the metric $d_{F}$ is not complete. Nevertheless he sketches a proof of the fact that $\left(P(N), \mathcal{T}\left(d_{F}\right)\right)$ is a Baire space, i.e., each of its non-null open sets is second category. Banach $[2$, p. 229] noted that $P(N)$ with the metric $d_{B}$ defined by

$$
d_{B}(s, t)=\sum_{i=1}^{\infty} \frac{1}{2^{i}} \frac{|s(i)-t(i)|+\left|s^{-1}(i)-t^{-1}(i)\right|}{1+|s(i)-t(i)|+\left|s^{-1}(i)-t^{-1}(i)\right|}
$$

for each $s$ and $t$ in $P(N)$ is a complete metric space and if one uses composition of functions as multiplication, $\left(P(N), \mathcal{T}\left(d_{B}\right)\right)$ is a topo-

Presented to the Society, November 26, 1954; received by the editors August 25, 1954.

1 This paper is from Chapter IV of the author's dissertation, On unconditional summability of sequences in semigroups with a topology. The Tulane University of Louisiana, August 1954. It was done under Contract N7-onr-434, Task Order III, Navy Department, Office of Naval Research.

${ }^{2}$ See, for example, $[4 ; 5 ; 6]$. In this connection the theorem of a recent note of A. Huber, Note on a theorem of Ostrowski, which appeared in these Proceedings, June, 1954 , is a special case of [4, Corollary 3.1]. 
logical group. Schreier and Ulam [7] have noted that $P(N)$ with the metric $d_{F^{\prime}}$ defined by $d_{F^{\prime}}(s, t)=d_{F}(s, t)+d_{F}\left(s^{-1}, t^{-1}\right)$ for each $s$ and $t$ in $P(N)$ is a complete metric space. One may easily verify that $d_{F}(s, t) \leqq d_{B}(s, t) \leqq d_{F^{\prime}}(s, t)$ for all $s$ and $t$ in $P(N)$. Also it is known that if $\left\{s_{n}\right\}$ is a sequence in $P(N)$ and $s \in P(N)$, then $\lim _{n \rightarrow \infty} d_{F}\left(s_{n}, s\right)$ $=0$ if and only if $\lim _{n \rightarrow \infty} d_{F}\left(s_{n}^{-1}, s^{-1}\right)=0$. It follows from these remarks and the definition of $d_{F^{\prime}}$ that if $\left\{s_{n}\right\}$ is a sequence in $P(N)$ and $s \in P(N)$, then $\lim _{n \rightarrow \infty} s_{n}=s$ with respect to one of the metrics $d_{F}$, $d_{B}$, and $d_{F^{\prime}}$ if and only if $\lim _{n \rightarrow \infty} s_{n}=s$ with respect to all of them. Therefore all three metrics induce the same topology on $P(N)$, i.e., $\mathcal{T}\left(d_{F}\right)=\mathcal{T}\left(d_{B}\right)=\mathcal{T}\left(d_{F^{\prime}}\right)$. Since $P(N)$ with the metric $d_{B}$ is a complete metric space it follows that $\left(P(N), \mathcal{T}\left(d_{B}\right)\right)$ is a Baire space and hence $\left(P(N), \mathcal{T}\left(d_{F}\right)\right)$ is a Baire space since $\mathcal{T}\left(d_{F}\right)=\mathcal{T}\left(d_{B}\right)$. This way of showing that $\left(P(N), \mathcal{T}\left(d_{F}\right)\right)$ is a Baire space differs from the way outlined by Agnew [1].

Still another metric, $d_{A}$, may be defined for $P(N)$ by setting $d_{A}(s, t)=0$ if $s$ and $t$ are the same permutation of $N$ and if $s$ and $t$ are distinct permutations of $N$ by setting $d_{A}(s, t)=1 / n$ where $n$ is the least natural number $i$ such that $s(i) \neq t(i)$. By a remark of Agnew [1], $\mathcal{T}\left(d_{F}\right)=\mathcal{T}\left(d_{A}\right)$. Because of its simplicity and its equivalence to $d_{F}$, the metric $d_{A}$ will be used in the sequel to verify statements about $\left(P(N), \tau\left(d_{F}\right)\right)$.

I wish to thank Professor B. J. Pettis for his helpful suggestions regarding this paper.

2. Lemmas for the main theorem. Suppose $Y$ is a topological space with a uniform topology, as defined in [9], determined by the uniformity $\left[V_{\alpha}: \alpha \in A\right]$. Suppose further that $\Lambda=[\lambda, \geqq]$ is a directed set and $X$ is a topological space and that $\left\{f_{\lambda}\right\}$ is a family of functions such that for each $\lambda \in \Lambda, f_{\lambda}$ is a function on $X$ to $Y$. An element $x$ of $X$ is a point of convergence of $\left\{f_{\lambda}\right\}$ if $\left\{f_{\lambda}(x)\right\}$ is a convergent net in $Y$. The set of points of convergence of $\left\{f_{\lambda}\right\}$ will be denoted by $C^{\prime}\left(f_{\lambda}\right)$. The defining property in the next definition was used by Osgood $[5, \mathrm{p}$. 161]. A point $x_{0} \in X$ is a point of uniform convergence of $\left\{f_{\lambda}\right\}$ if there is $y \in Y$ such that for each $\alpha \in A$ there is an open set $G_{\alpha}$ containing $x_{0}$ and there is $\lambda_{\alpha} \in \Lambda$ with $f_{\lambda}(x) \in V_{\alpha}(y)$ for all $x \in G_{\alpha}$ and $\lambda \geqq \lambda_{\alpha}$. The set of points of uniform convergence of $\left\{f_{\lambda}\right\}$ will be denoted by $C_{u}^{\prime}\left(f_{\lambda}\right)$. A point $x_{0}$ in $X$ is a Cauchy point of $\left\{f_{\lambda}\right\}$ [6] if and only if $\left\{f_{\lambda}\left(x_{0}\right)\right\}$ is a Cauchy net in $Y$. The set of Cauchy points of $\left\{f_{\lambda}\right\}$ will be denoted by $C\left(f_{\lambda}\right)$. A point $x_{0}$ in $X$ is a uniform Cauchy point of $\left\{f_{\lambda}\right\}$ [6] if and only if to each $\alpha \in A$ there corresponds $\lambda_{\alpha} \in \Lambda$ and an open set $G$ containing $x_{0}$ such that $f_{\lambda}(x) \in V_{\alpha}\left(f_{\lambda \alpha}(x)\right)$ for all $\lambda \geqq \lambda_{\alpha}$ and all $x$ in $G$. 
Lemma 1. The relations $C^{\prime}\left(f_{\lambda}\right) \subset C\left(f_{\lambda}\right)$ and $C_{u}^{\prime}\left(f_{\lambda}\right) \subset C_{u}\left(f_{\lambda}\right)$ always hold. If $C^{\prime}\left(f_{\lambda}\right)=C\left(f_{\lambda}\right)$ and each $f_{\lambda}$ is continuous then $C_{u}^{\prime}\left(f_{\lambda}\right)=C_{u}\left(f_{\lambda}\right)$.

Proof. The first statement of the theorem is evident. Because of it the second statement will follow if one shows that $C_{u}\left(f_{\lambda}\right) \subset C_{u}^{\prime}\left(f_{\lambda}\right)$. Assume the hypothesis of the second statement in the theorem and suppose $x_{0} \in C_{u}\left(f_{\lambda}\right)$. Let $\alpha$ be an element of $A$. Then there is $\beta \in A$ such that if $p, q$, and $r$ are elements of $Y$ such that $p \in V_{\beta}(r)$ and $q \in V_{\beta}(r)$, then $p \in V_{\alpha}(q)$. Since $x_{0} \in C_{u}\left(f_{\lambda}\right)$ it follows from [6, Lemma 1] that $x_{0} \in C\left(f_{\lambda}\right) \cap C_{a}\left(f_{\lambda}\right)$ where $C_{a}\left(f_{\lambda}\right)$ is the set of points of almost equicontinuity of $\left\{f_{\lambda}\right\}$. By definition $x_{0} \in C_{a}\left(f_{\lambda}\right)$ means that for arbitrary $\beta \in A$ (and hence for the $\beta$ of the preceding sentence) there is $\lambda_{\beta} \in \Lambda$ and an open set $G \ni x_{0}$ such that $f_{\lambda}(x) \in V_{\beta}\left(f_{\lambda}\left(x_{0}\right)\right)$ for all $\lambda \geqq \lambda_{\beta}$ and $x \in G$. Since $x_{0} \in C\left(f_{\lambda}\right)=C^{\prime}\left(f_{\lambda}\right)$ there is $y_{0} \in Y$ such that the net $\left\{f_{\lambda}\left(x_{0}\right)\right\}$ is convergent to $y_{0}$. Next there is $\gamma \in A$ such that if $s, t$, and $u$ are elements of $Y$ and $s \in V_{\gamma}(u)$ and $t \in V_{\gamma}(u)$, then $s \in V_{\beta}(t)$; also by symmetry of this condition $t \in V_{\beta}(s)$. Because $\left\{f_{\lambda}\left(x_{0}\right)\right\}$ is convergent to $y_{0}$ there is $\lambda_{\gamma} \in \Lambda$ such that if $\lambda \geqq \lambda_{\gamma}$ then $f_{\lambda}\left(x_{0}\right) \in V_{\gamma}\left(y_{0}\right)$. Choose some $\lambda_{\alpha} \in \Lambda$ such that $\lambda_{\alpha} \geqq \lambda_{\beta}$ and $\lambda_{\alpha} \geqq \lambda_{\gamma}$ and suppose $\lambda \in \Lambda$ and $\lambda \geqq \lambda_{\alpha}$. Then one has that $f_{\lambda}\left(x_{0}\right) \in V_{\gamma}\left(y_{0}\right)$ and $y_{0} \in V_{\gamma}\left(y_{0}\right)$ so $f_{\lambda}\left(x_{0}\right) \in V_{\beta}\left(y_{0}\right)$ and $y_{0} \in V_{\beta}\left(f_{\lambda}\left(x_{0}\right)\right)$. Now if $x$ is an element of $G$ it follows that $f_{\lambda}(x) \in V_{\beta}\left(f_{\lambda}\left(x_{0}\right)\right)$ since $\lambda \geqq \lambda_{\beta}$. Hence, $f_{\lambda}(x) \in V_{\beta}\left(f_{\lambda}\left(x_{0}\right)\right)$ and $y_{0} \in V_{\beta}\left(f_{\lambda}\left(x_{0}\right)\right)$ so $f_{\lambda}(x) \in V_{\alpha}\left(y_{0}\right)$. It has been shown that $x_{0} \in C_{u}^{\prime}\left(f_{\lambda}\right)$. The next lemma is a special case of part of $[4$, Theorem 3$]$.

Lemma 2. Suppose $X$ is a topological space and $Y$ is a pseudo-metric space and $\left\{f_{n}\right\}$ is a sequence of continuous functions on $X$ to $Y$. If $C\left(f_{n}\right)$ is second category so is $C_{u}\left(f_{n}\right)$.

Throughout the remainder of this paper $\mathcal{F}$ will denote the family of non-null, finite subsets of the natural numbers $N$. Also let $[1, n]$ $=[k \in N: 1 \leqq k \leqq n]$; and for any set $S$ let $\varnothing$ stand for the void subset of $S$. The topology $\mathcal{T}\left(d_{F}\right)$ will be used for $P(N)$ throughout the paper.

LEMMA 3. If $X$ is an additively written semigroup with a topology and $s=\{s(i)\}$ is a sequence in $X$, then for each $n \in N$ the function $\sigma[n, s]$ defined on $P(N)$ to $X$ by $\sigma[n, s](t)=\sum_{i=1}^{n} s(t(i))$ for each $t \in P(N)$ is continuous.

Proof. Suppose $t \in P(N)$ and $H$ is an open subset of $X$ containing $\sigma[n, s](t)$. If $t^{\prime} \in P(N)$ and $d_{A}\left(t, t^{\prime}\right)<1 / n+1$, then $t(i)=t^{\prime}(i)$ for $i \in[1, n]$ so $\sigma[n, s]\left(t^{\prime}\right)=\sigma[n, s](t) \in H$. Hence, $\sigma[n, s]$ is continuous. 
The following well known fact about permutation is also needed.

Lemma 4. Suppose $t_{0} \in P(N), n \in N$, and $D=\left\{d_{j}\right\}_{j=1}^{p}$ is a finite set of $p>0$ positive integers and $t_{0}([1, n]) \cap D=\varnothing$. Then there is an $n^{\prime}>n$ and $t \in P(N)$ such that $t(i)=t_{0}(i)$ for all $i \in[1, n] ; t(n+j)=d_{j}$ for all $j \in[1, p]$; and $t(i)=t_{0}(i)$ for $i>n^{\prime}$.

\section{The main theorem.}

THEOREM 1. Suppose $s=\{s(i)\}$ is a sequence in a commutative semigroup $X$ with a topology, and suppose that $\psi$ is a real valued continuous function on $X$ such that:

(1) $\psi(x+y) \leqq \psi(x)+\psi(y)$ for all $x$ and $y$ in $X$.

(2) $\psi(y) \leqq \psi(x+y)+|\psi(x)|$ for all $x$ and $y$ in $X$.

Let

$$
A=\left[t \in P(N): \sup \left\{\psi\left(\sum_{i \in D} s(t(i))\right): D \in \mathcal{F}\right\}<+\infty\right]
$$

and let

$$
B=\left[t \in P(N): \sup \left\{\psi\left(\sum_{i=1}^{n} s(t(i))\right): n \in N\right\}<+\infty\right] .
$$

Then the following conclusions are valid:

(i) The set $B$ is an $F_{\sigma}$ subset of $\left(P(N), \mathcal{O}\left(d_{F}\right)\right)$.

(ii) If $A$ is non-null then $A=B=P(N)$ and there is a real number $K$ such that $\sup \left[\psi\left(\sum_{i \in D} s(t(i))\right): D \in \mathcal{F}\right.$ and $\left.t \in P(N)\right] \leqq K$.

(iii) If $A$ is null then $B$ is first category in $\left(P(N), \mathcal{T}\left(d_{F}\right)\right)$ and $P(N) \backslash B$ is a second category everywhere dense $G_{\delta}$ subset of $P(N)$.

(iv) If $B$ is a second category subset of $\left(P(N), \mathcal{T}\left(d_{F}\right)\right)$ then $A=B$ $=P(N)$.

(v) The set $P(N) \backslash B$ is residual in $\left(P(N), \mathcal{G}\left(d_{F}\right)\right)$ if and only if it is non-null.

Proof. For each $n \in N$ define a function $F_{n}$ on $P(N)$ into the reals by $F_{n}(t)=\max \left[\psi\left(\sum_{i=1}^{j} s(t(i)): j \in[1, n]\right]\right.$ for each $t \in P(N)$. By Lemma 3, for each $j \in N$ the function $S_{j}$ defined on $P(N)$ to $X$ by $S_{j}(t)=\sum_{i=1}^{j} s(t(i))$ for each $t \in P(N)$ is continuous. Then $\psi S_{j}$ is continuous on $P(N)$ to the reals for each $j \in N$ since it is the composition of continuous functions. Therefore since $F_{n}(t)=\max \left[\psi\left(S_{j}(t)\right)\right.$ : $j \in[1, n]]$ for each $t \in P(N), F_{n}$ is continuous for each $n \in N$. Now for each $t \in P(N)$ the sequence $\left\{F_{n}(t)\right\}$ is a nondecreasing sequence of real numbers. Hence, $C^{\prime}\left(F_{n}\right)$, the convergence set of the nondecreasing sequence of continuous functions $\left\{F_{n}\right\}$, is an $F_{\sigma}$ subset of $P(N)$. 
Furthermore, it is clear that $t \in B$ if and only if $\lim _{n \rightarrow \infty} F_{n}(t)$ exists and is finite, i.e., $B=C^{\prime}\left(F_{n}\right)$. This proves that $B$ is an $F_{\sigma}$ subset of $P(N)$ and hence that $P(N) \backslash B$ is a $G_{\delta}$ subset of $P(N)$.

It is obvious that $A \subset B$. Suppose $A$ is non-null. Then there is $t_{0} \in P(N)$ and a finite real number $K$ such that

$$
\sup \left[\psi\left(\sum_{i \in D} s\left(t_{0}(i)\right)\right): D \in \mathcal{F}\right] \leqq K .
$$

Let $t$ and $D$ be arbitrary elements of $P(N)$ and $\mathcal{F}$ respectively. Since $t$ and $t_{0}$ are elements of $P(N)$ there is $D_{0} \in \mathcal{F}$ such that $t(D)=t_{0}\left(D_{0}\right)$. Therefore $\psi\left(\sum_{i \in D} s(t(i))\right)=\psi\left(\sum_{i \in D_{0}} s\left(t_{0}(i)\right)\right) \leqq K$. This completes the proof of (ii).

Statement (iii) will now be verified. Suppose $B=C^{\prime}\left(F_{n}\right)$ is a second category subset of $P(N)$. By Lemma 1 and the completeness of the reals it follows that $C^{\prime}\left(F_{n}\right)=C\left(F_{n}\right)$ and $C_{u}^{\prime}\left(F_{n}\right)=C_{u}\left(F_{n}\right)$. Since $C^{\prime}\left(F_{n}\right)=C\left(F_{n}\right)$ is second category in $P(N)$ and $\left\{F_{n}\right\}$ is a sequence of continuous functions it follows by Lemma 2 that $C_{u}\left(F_{n}\right)=C_{u}^{\prime}\left(F_{n}\right)$ is also second category in $P(N)$. Hence there exists $t_{0} \in C_{u}^{\prime}\left(F_{n}\right)$. Therefore $\epsilon=1$ implies there is $n_{0} \in N$ such that if $t \in G \equiv\left[t^{\prime} \in P(N)\right.$ : $\left.d_{A}\left(t_{0}, t^{\prime}\right)<1 / n_{0}\right]$ and $n \geqq n_{0}$ then $F_{n}(t)<\left(\lim _{n \rightarrow \infty} F_{n}\left(t_{0}\right)\right)+1$. Because of the definition of $d_{A}$ this can be rephrased as follows:

If $t \in P(N)$ and $t(i)=t_{0}(i)$ for $i \in\left[1, n_{0}\right]$ then

$$
\psi\left(\sum_{i=1}^{j} s(t(i))\right)<\lim _{n \rightarrow \infty} F_{n}\left(t_{0}\right)+1 \quad \text { for every } j \text { in } N .
$$

It will be shown by considering four cases that the identity of $P(N)$ is an element of $A$. Let $D$ be an arbitrary but fixed element of $\mathcal{F}$. Let $K \equiv \max \left(\sum_{i=1}^{n_{0}}\left|\psi\left(s\left(t_{0}(i)\right)\right)\right|, \lim _{n \rightarrow \infty} F_{n}\left(t_{0}\right)+1\right)$. In case

$$
D \subset t_{0}([1, n]) \text {, }
$$

using property (1) of $\psi$ one has

$$
\left.\psi\left(\sum_{i \in D} s(i)\right) \leqq \sum_{i \in D} \psi(s(i)) \leqq \sum_{i \in D} \mid \psi(s(i))\right)\left|\leqq \sum_{i=1}^{n_{0}}\right| \psi\left(s\left(t_{0}(i)\right)\right) \mid \leqq K .
$$

Now consider the case where $t_{0}\left(\left[1, n_{0}\right]\right)$ is a proper subset of $D$. Let $\left\{d_{j}\right\}_{j=1}^{p}$ denote the elements of $D \backslash t_{0}\left(\left[1, n_{0}\right]\right)$. Then by Lemma 4 there is $t \in p(N)$ such that $t(i)=t_{0}(i)$ for $i \in\left[1, n_{0}\right]$ and $t\left(n_{0}+j\right)=d_{j}$ for $j \in[1, p]$. Using property $\left(^{*}\right)$ it follows that

$$
\psi\left(\sum_{i \in D} s(i)\right)=\psi\left(\sum_{i=1}^{n_{0}+p} s(t(i))\right)<K
$$


which finishes the verification of this case. Next suppose that $t_{0}\left(\left[1, n_{0}\right]\right) \cap D$ is the null set. In this case, using property (2) of $\psi$, it follows that

$$
\begin{gathered}
\psi\left(\sum_{i \in D} s(i)\right) \leqq \psi\left(\left(\sum_{i=1}^{n_{0}} s\left(t_{0}(i)\right)\right)+\sum_{i \in D} s(i)\right) \\
+\left|\psi\left(\sum_{i=1}^{n_{0}} s\left(t_{0}(i)\right)\right)\right| .
\end{gathered}
$$

Let $d_{1}, d_{2}, \cdots, d_{p}$ denote any ordering of the elements of $D$. By Lemma 2 there is $t \in P(N)$ such that $t(i)=t_{0}(i)$ for $i \in\left[1, n_{0}\right]$ and $t\left(n_{0}+j\right)=d_{j}$ for $j \in[1, p]$. For such a permutation $t$ the equality $\left(\sum_{i=1}^{n_{0}} s\left(t_{0}(i)\right)\right)+\sum_{i \in D} s(i)=\sum_{i=1}^{n_{0}+p} s(t(i))$ holds. From this together with proposition $\left({ }^{*}\right)$ it follows that the first term on the righthand side of the preceding inequality is no greater than $K$. Using property (1) of $\psi$ the second term is no greater than $K$ so $\psi\left(\sum_{i \in D} s(i)\right) \leqq 2 K$ in this case. Finally suppose both

$$
t_{0}\left(\left[1, n_{0}\right]\right) \cap D \text { and } F \equiv D \backslash t_{0}\left(\left[1, n_{0}\right]\right)
$$

are non-null. Let $E \equiv t_{0}\left(\left[1, n_{0}\right]\right) \backslash D$. Set $y=\sum_{i \in D} s(i)$ and $x=\sum_{i \in E} s(i)$. By property (2) of $\psi$ one then has $\psi(y) \leqq \psi(x+y)$ $+|\psi(x)|$. Note that $x+y=\sum_{i=1}^{n_{0}} s\left(t_{0}(i)\right)+\sum_{i \in F} s(i)$. Using Lemma 4 there is $t_{1} \in P(N)$ and $n \in N$ such that $t_{1}(i)=t_{0}(i)$ for $i \in\left[1, n_{0}\right]$ and $\sum_{i=1}^{n} s\left(t_{1}(i)\right)=x+y$. Therefore by proposition $\left({ }^{*}\right), \psi(x+y) \leqq K$. That $|\psi(x)| \leqq K$ follows from the inequality $|\psi(x)|=\left|\psi\left(\sum_{i \in E} s(i)\right)\right|$ $\leqq \sum_{i=1}^{n_{0}}\left|\psi\left(s\left(t_{0}(i)\right)\right)\right|$ and the definition of $K$. Hence, in this case also $\psi(y) \leqq 2 K$.

To verify statement (iv) suppose that $B$ is second category. Then by (iii), $A$ is non-null so by (ii), $A=B=P(N)$.

To verify statement (v) suppose that $P(N) \backslash B$ is residual in $P(N)$. Since $P(N)$ is second category in itself, $P(N) \backslash B$ is also second category in $P(N)$ so it is non-null. Conversely, if $P(N) \backslash B$ is non-null then $B$ is first category in $P(N)$, i.e., $P(N) \backslash B$ is residual. If $B$ were not first category then $B$ would equal $P(N)$ by (iv) which contradicts the supposition that $P(N) \backslash B$ is non-null.

4. Applications. N. Bourbaki [3] has shown that conditions (iii) and $(\mathrm{v})$ in the following theorem are equivalent if $X$ is any abelian topological group.

THEOREM 2. Suppose $(X,+, \mathcal{G}(d))$ is a complete commutative semigroup with a topology $\mathcal{T}(d)$ determined by the pseudo metric d. Let $s=\{s(i)\}$ denote a sequence in $X$ and for each $n \in N$ let $\sigma(n, s)$ denote 
the function on $P(N)$ to $X$ defined in Lemma 3. The following conditions on $s$ are pairwise equivalent.

(i) $C^{\prime}(\sigma[n, s]) \equiv\left[t \in P(N)\right.$ : the sequence $\left\{\sum_{i=1}^{n} s(t(i))\right\}_{n=1}^{\infty}$ is convergent $]$ is second category in $\left(P(N), \mathcal{T}\left(d_{F}\right)\right)$.

(ii) $C_{u}^{\prime}(\sigma[n, s]) \neq \varnothing$.

(iii) There is an $x \in X$ such that to each $\epsilon>0$ there corresponds $F_{\epsilon} \in \mathcal{F}$ such that if $F \in \mathcal{F}$ and $F \supset F_{\epsilon}$, then $d\left(\sum_{i \in F} s(i), x\right)<\epsilon$.

(iv) $C_{u}^{\prime}(\sigma[n, s])=P(N)$.

(v) $C^{\prime}(\sigma[n, s])=P(N)$, i.e., the series $\sum_{i=1}^{\infty} s(i)$ is unconditionally convergent.

Proof. Since $P(N)$ is second category, (v) obviously implies (i).

Next assume that $C^{\prime}(\sigma[n, s])$ is second category. Since $X$ is complete, $C^{\prime}(\sigma[n, s])=C(\sigma[n, s])$ so, by Lemma $1, C_{u}^{\prime}(\sigma[n, s])$ $=C_{u}(\sigma[n, s])$. Since $C^{\prime}(\sigma[n, s])$ is second category in $P(N)$ it follows using Lemma 2 that $C_{u}^{\prime}(\sigma[n, s])$ is second category in $P(N)$ which proves that (i) implies (ii) since any second category set is non-null.

Suppose that $C_{u}^{\prime}(\sigma[n, s]) \neq \varnothing$. Therefore there is $t_{0} \in P(N)$ with these properties:

(*) There exists $x \in X$ such that to each $\epsilon>0$ there corresponds $n_{\epsilon} \in N$ such that if $t \in P(N)$ and $t(i)=t_{0}(i)$ for $i \in\left[1, n_{\epsilon}\right]$, then $d\left(\sum_{t=1}^{n} s(t(i)), x\right)<\epsilon$ for all $n \geqq n_{\epsilon}$.

To show that property $\left(^{*}\right)$ implies (iii) let $\epsilon>0$ be given. Let $n_{\epsilon}$ be the natural number whose existence is assured by $\left(^{*}\right)$ and let $F_{\epsilon}=\left[t_{0}(i): i \in\left[1, n_{\epsilon}\right]\right]$. Suppose $F \in \mathcal{F}$ and $F \supset F_{\epsilon}$. Let $n_{\epsilon}+p$ denote the number of elements in $F$. By Lemma 4 there is $t \in P(N)$ such that $t(i)=t_{0}(i)$ for $i \in\left[1, n_{\epsilon}\right]$ and $t\left(\left[1, n_{\epsilon}+p\right]\right)=F$. Using $\left(^{*}\right)$ it follows that $d\left(\sum_{i \in F} s(i), x\right)=d\left(\sum_{i=1}^{n \epsilon+p} s(t(i)), x\right)<\epsilon$. This proves that (ii) implies (iii).

Next suppose that (iii) holds, that $t_{0} \in P(N)$, and that $\epsilon>0$. Let $F_{\epsilon}$ denote an element of $\mathcal{F}$ satisfying statement (iii). Choose $n_{\epsilon}$ large enough so that $t_{0}\left(\left[1, n_{\epsilon}\right]\right) \supset F_{\epsilon}$. If $t \in P(N)$ and $t(i)=t_{0}(i)$ for $i \in\left[1, n_{\epsilon}\right]$ and $n \geqq n_{\epsilon}$, then $t([1, n]) \supset F_{\epsilon}$ so $d\left(\sum_{t=1}^{n} s(t(i)), x\right)<\epsilon$. This proves that $t_{0} \in C_{u}^{\prime}(\sigma[n, s])$ and hence that (iii) implies (iv).

Obviously (iv) implies (v). This completes the proof of Theorem 2.

TheOREM 3. Suppose $(X,+, \mathcal{T}(d))$ is a commutative semigroup with $a$ zero $\theta$ and a topology $\mathcal{\tau}(d)$ determined by a pseudo metric $d$ such that $d(x, y)=d(z+x, z+y)$ for all $x, y$, and $z$ in $X$. If $s=\{s(i)\}$ is a sequence in $X$ such that $\left[t \in P(N): \sup \left\{d\left(\sum_{i=1}^{n} s(t(i)), \theta\right): n \in N\right\}<\infty\right]$ is a second category subset of $\left(P(N), \mathcal{T}\left(d_{F}\right)\right)$, then there is a real number $K$ such that $\sup \left[d\left(\sum_{i \in D} s(t(i)), \theta\right): D \in \mathcal{F}, t \in P(N)\right] \leqq K$.

Proof. Define a function $\psi$ on $X$ into the reals by $\psi(x)=d(x, \theta)$ for 
each $x \in X$. All hypotheses of Theorem 1 are satisfied as can easily be verified, and hence from (iv) and (ii) of that theorem the conclusion follows.

Corollary 3.1. If $X$ is a weakly complete Banach space and $\sum_{i=1}^{\infty} x(i)$ is a series whose terms are elements of $X$, then $\sum_{i=1}^{\infty} x(i)$ is unconditionally convergent if and only if

$$
B \equiv\left[t \in P(N): \sup \left\{\left\|\sum_{i=1}^{n} x(t(i))\right\|: n \in N\right\}<\infty\right]
$$

is second category in $\left(P(N), \mathcal{T}\left(d_{F}\right)\right)$.

Proof. If $\sum_{i=1}^{\infty} x(i)$ is unconditionally convergent, then for each $t \in P(N)$ the sequence $\left\{\sum_{i=1}^{n} x(t(i))\right\}_{n=1}^{\infty}$ is a Cauchy sequence so it is bounded in $X$. Hence $B=P(N)$ which is second category. Conversely suppose $B$ is second category in $P(N)$. Using the usual metric $d(x, y)=\|x-y\|$ for each $x$ and $y$ in $X$ it is immediate from the conclusion of Theorem 3 , taking $t$ to be the identity permutation, that sup $\left[\left\|\sum_{i \in F} x(i)\right\|: F \in \mathcal{F}\right]<\infty$ and it is well known that this condition implies that $\sum_{i=1}^{\infty} x(i)$ converges unconditionally since $X$ is a weakly complete Banach space.

Agnew [ 1 ] noted that if $\sum_{i=1}^{\infty} z(i)$ is a convergent series of complex numbers for which $\sum_{i=1}^{\infty}\|z(i)\|=+\infty$ then the set of $t \in P(N)$ for which $\sum_{i=1}^{\infty} z(t(i))$ has bounded partial sums is a set of the first category. The following result which includes his is obtained by a different proof.

Corollary 3.2. If $s=\{s(i)\}$ is a sequence in a Banach space $X$ with finite dimension and $\sum_{i=1}^{\infty}\|s(i)\|=+\infty$ then

$$
E \equiv\left[t \in P(N): \sup \left\{\left\|\sum_{i=1}^{n} s(t(i))\right\|: n \in N\right\}=+\infty\right]
$$

is a residual everywhere dense $G_{\delta}$ subset of $\left(P(N), \mathcal{T}\left(d_{F}\right)\right)$.

Proof. Let $\psi(x)=\|x\|$. By (i) of Theorem $1, E$ is a $G_{\delta}$ subset of $P(N)$. Suppose $E$ is not residual, i.e., suppose $P(N) \backslash E$ is second category. Then using Theorem 3 there exists a finite number $K$ such that sup $\left[\left\|\sum_{i \in F} s(i)\right\|: F \in \mathcal{F}\right] \leqq K$. It is well known that, for a Banach space with finite dimension, the above boundedness condition implies that the series $\sum_{i=1}^{\infty} s(i)$ converges unconditionally. This however is a contradiction because unconditional convergence implies absolute convergence when $X$ has finite dimension. Therefore $E$ is residual and hence since it is a residual $G_{\delta}$ subset of a Baire space it is everywhere dense. 
If $s=\{s(i)\}$ is a sequence of real numbers let $E_{+\infty}(s)=[t \in P(N)$ : $\left.\lim \sup _{n} \sum_{i=1}^{n} s(t(i))=+\infty\right]$ and let

$$
E_{-\infty}(s)=\left[t \in P(N): \lim _{n} \inf \sum_{i=1}^{n} s(t(i))=-\infty\right] .
$$

Agnew [1] and Sengupta [8] have proved the following theorem:

THEOREM 4. If $s=\{s(i)\}$ is a sequence of real numbers such that $\lim \sum_{i=1}^{n} s(i)$ exists and $\sum_{i=1}^{\infty}|s(i)|=+\infty$, then $E_{+\infty}(s) \cap E_{-\infty}(s)$ is a residual everywhere dense $G_{\delta}$ subset of $\left(P(N), \mathcal{T}\left(d_{F}\right)\right)$.

The hypothesis of Theorem 4 is sufficient but not necessary for its conclusion. For example it follows from Theorem 6 that if $s$ is defined at each $i \in N$ by $s(i)=1^{i-1}$, then the conclusion of Theorem 4 holds for $s$. However, $\sum_{i=1}^{\infty} s(i)$ is not a convergent series. Part (iii) of Theorem 5 gives one necessary and sufficient condition for the conclusion of Theorem 4 and Theorem 6 gives another.

THEOREM 5. The following statements are valid for any sequence $s=\{s(i)\}$ of real numbers.

(i) The set $E_{+\infty}(s)$ is a residual everywhere dense $G_{\delta}$ subset of $(P(N)$, $\left.\mathcal{T}\left(d_{F}\right)\right)$ if and only if $E_{+\infty}(s)$ is non-null.

(ii) The set $E_{-\infty}(s)$ is a residual everywhere dense $G_{\delta}$ subset of $P(N)$ if and only if $E_{-\infty}(s)$ is non-null.

(iii) The set $E_{+\infty}(s) \cap E_{-\infty}(s)$ is a residual everywhere dense $G_{\delta}$ subset of $P(N)$ if and only if $E_{+\infty}(s)$ is non-null and $E_{-\infty}(s)$ is non-null.

Proof. Let $\psi$ denote the identity map of the additive group of the real numbers with its usual topology. Note that $\psi$ satisfies the hypothesis of Theorem 1 . Using $B$ as defined in that theorem it is clear that $B=\left[t \in P(N): \lim \sup _{n} \sum_{i=1}^{n} s(t(i))<\infty\right]$ so $E_{+\infty}(s)$ $=P(N) \backslash B$. By (i) of Theorem $1, E_{+\infty}(s)$ is a $G_{\delta}$ subset of $P(N)$ and by (v) of Theorem $1, E_{+\infty}(s)$ is residual in $P(N)$ if and only if it is nonnull. Since $E_{+\infty}(s)$ is a $G_{\delta}$ subset of the Baire space $P(N)$ it is everywhere dense in $P(N)$ when and only when it is residual in $P(N)$. This proves statement (i). Statement (ii) follows from (i) and the fact that $E_{-\infty}(s)=E_{+\infty}(-s)$ where $-s$ is defined at each $i \in N$ by $-s(i)$. Since $P(N)$ is second category in itself, if $E_{+\infty}(s) \cap E_{-\infty}(s)$ is residual in $P(N)$ then it is non-null so $E_{+\infty}(s)$ and $E_{-\infty}(s)$ are non-null. Conversely if $E_{+\infty}(s)$ and $E_{-\infty}(s)$ are non-null then by (i) and (ii) each is a residual $G_{\delta}$ subset of $P(N)$ and therefore so is their intersection. Hence their intersection is everywhere dense since $P(N)$ is a Baire space.

For the sake of brevity the following fairly evident lemma is stated without proof. 
Lemma 5. Suppose $s=\{s(i)\}$ is a sequence of real numbers. Then, $E_{+\infty}(s)$ is non-null if and only if $\sum_{i=1}^{\infty} s\left(t_{0}(i)\right)=+\infty$ where $\left\{s\left(t_{0}(i)\right)\right\}$ is the subsequence of non-negative terms of $s$. Also, $E_{-\infty}(s)$ is non-null if and only if $\sum_{i=1}^{\infty} s\left(t_{1}(i)\right)=-\infty$ where $\left\{s\left(t_{1}(i)\right)\right\}$ is the subsequence of negative terms of $s$.

THEOREM 6. If $s=\{s(i)\}$ is a sequence of real numbers, then $E_{+\infty}(s)$ $\cap E_{-\infty}(s)$ is a residual everywhere dense $G_{\delta}$ subset of $\left(P(N), \mathcal{T}\left(d_{F}\right)\right)$ if and only if $\sum_{i=1}^{\infty} s\left(t_{0}(i)\right)=+\infty$ and $\sum_{i=1}^{\infty} s\left(t_{1}(i)\right)=-\infty$ where $\left\{s\left(t_{0}(i)\right)\right\}$ and $\left\{s\left(t_{1}(i)\right)\right\}$ are the subsequences of non-negative and negative terms of $s$ respectively.

Proof. The conclusion follows immediately from (iii) of Theorem 5 together with Lemma 5.

If $s=\{s(i)\}$ is a sequence of real numbers such that $\lim _{n \rightarrow \infty} \sum_{i=1}^{n} s(i)$ exists and $\sum_{i=1}^{\infty}|s(i)|=+\infty$ then it follows, using the notation of Theorem 6, that $\sum_{i=1}^{\infty} s\left(t_{0}(i)\right)=+\infty$ and $\sum_{i=1}^{\infty} s\left(t_{1}(i)\right)=-\infty$. Hence, the Agnew-Sengupta theorem, Theorem 4, is a corollary of Theorem 6.

\section{REFERENCES}

1. R. P. Agnew, On rearrangements of series, Bull. Amer. Math. Soc. vol. 46 (1940) pp. 797-799.

2. S. Banach, Thérie des operations lineaires, Warsaw, 1932, $7+254 \mathrm{pp}$.

3. N. Bourbaki, Eléments de mathématique, Topologie générale, $2 \mathrm{~d}$ ed., Chap. III, Paris, 1951, 158 pp.

4. W. L. Gordon and C. W. McArthur, A theorem on uniform Cauchy points, Amer. J. Math. vol. 74 (1952) pp. 764-767.

5. W. F. Osgood, Non-uniform convergence and the integration of series term by term, Amer. J. Math. vol. 19 (1897) pp. 155-190.

6. B. J. Pettis, Uniform Cauchy points and points of equicontinuity, Amer. J. Math. vol. 73 (1951) pp. 602-614.

7. O. Schreier and S. Ulam, Über die Permutationsgruppe der naturalichen Zahlenfolge, Studia Math. vol. 4 (1933) pp. 134-141.

8. H. M. Sengupta, On rearrangements of series, Proc. Amer. Math. Soc. vol. 1 (1950) pp. 71-75.

9. A. Weil, Sur les espaces d̀ structure uniforme et sur la topologie générale, Actualités Scientifiques et Industrielles, no. 551, Paris, Hermann, 1938, 39 pp.

The Tulane University of Louisiana and

Alabama Polytechnic Institute 